\title{
EQUIVALÊNCIA DE ESTÍMULOS E INSTRUÇÃO PROGRAMADA: FORMAÇÃO DE CLASSES COM ESTÍMULOS FRACIONÁRIOS
}

\author{
Antônio Carlos Godinho dos Santos* Prof. PUC- GO \\ acgodinhos@yahoo.com.br \\ Lorismario Ernesto Simonassi* Prof. PUC- GO \\ Rafaela Ferreira de Queiroz**A Aluna PUC- GO \\ Fabíola Belém Pacheco** Aluna PUC- GO \\ Maiara Maia de Santana** Aluna PUC- GO \\ Maíra Ribeiro Magri** Aluna PUC-GO \\ Gustavo Henrique Heime de Melo** Aluno PUC- GO
}

\begin{abstract}
RESUMO: Este trabalho estudou o efeito do paradigma de equivalência de estímulos e de instruções no ensino de frações. Cinco alunos com baixo desempenho em teste de múltipla escolha com problemas fracionários solucionaram problemas por meio de instrução programada e passaram pelo teste das relações derivadas (GIP). Cinco escolheram frações numéricas na presença de modelos pictóricos ou numéricos em tarefa de pareamento com o modelo (GEQ) e cinco fizeram ambas as tarefas (GIPEC). Resultados mostraram formação classes de equivalência de três membros com estímulos fracionários pictóricos e numéricos e decréscimos na porcentagem de acertos no teste de expansão das classes. Nos testes de múltipla escolha houve aumentos sistemáticos no número de acertos para os três grupos, em especial para o GIPEC.
\end{abstract}

Palavras-chave: Equivalência de frações. Conceito de proporção. Equivalência de estímulos.

\section{STIMULUS EQUIVALENCE AND PROGRAMED INSTRUCTIONS: CLASSES FORMATION WITH FRACTION STIMULUS}

\begin{abstract}
In this research the stimulus matching paradigm was applied to the teachinglearning of fractions. Five students with low performance on multiple choice tests with fraction problems, solved problems using programed instructions and then were submitted to the derivative relations test (GIP). Five chose numeric fractions in the presence of pictorial or numerical models in matching to the model tasks (GEQ) and five did both (GIPEC). Results show the formation of equivalency classes in three students with pictorial and numerical fractions stimuli, and a decrease in the percentage of hits on the test of expansion of classes. On the multiple choice questions there was a systematic increase in the number of hits in all three groups, but especially GIPEC.
\end{abstract}

Keywords: Equivalence of fractions. The concept of proportion. Stimuli equivalence. 


\section{(2ibnerarius Refiectionis: =-

$$
\text { v.11, n. } 1,2015
$$

\section{INTRODUÇÃO}

O paradigma de equivalência de estímulos proposto por Sidman e Tailby (1982) é mais um modelo que permite explicar como respostas que não foram ensinadas diretamente são aprendidas. Segundo esse modelo, o treino de algumas relações condicionais entre estímulos arbitrários pode originar relações denominadas como simétricas, transitivas e equivalentes originando uma classe de estímulos equivalentes. Uma vez formada uma classe, pode ocorrer sua expansão pelo treino direto entre um de seus membros e um novo estímulo (SIDMAN, KIRK; WILSSON-MORRIS, 1985; SAUNDERS, WACHTER; SPRADLIN, 1988).

Analistas do comportamento consideram a possibilidade de compreender a aquisição de conceitos matemáticos a partir da formação de classes de estímulos equivalentes (CARMO, 2000, DE ROSE, 2010) e utilizar o modelo como alternativa para o conceito de proporção, uma vez que é frequente alunos do início do ensino fundamental demonstrarem dificuldades para compreender o conceito de magnitude relativa entre frações (CARRAHER, CARRAHER; SCHLIEMANN, 1993; SANTOS, 1999). É comum, por exemplo, que alunos não reconheçam que as frações 1/3, 2/6 e 3/9 representem um mesmo valor quantitativo e constituam uma classe de equivalência ou um conceito.

Alguns estudos analítico-comportamentais investigaram a formação de classes de equivalência ou conceitos com estímulos fracionários a partir do paradigma de equivalência de estímulos. Linch e Cuvo (1995) estudaram a formação de classes de estímulos entre números fracionários e decimais. No trabalho de Santos, Cameschi e Hanna (2009) os alunos aprenderam a escolher frações numéricas correspondentes aos modelos pictóricos ou numéricos equivalentes. De forma geral, os resultados evidenciaram a formação de classes de equivalência entre os estímulos fracionários pictóricos e numéricos, mas desempenhos limitados nos testes de generalização e de expansão das classes.

Embora existam questionamentos antigos e recentes na literatura quanto à utilidade da regra de três como procedimento para ensinar o conceito de proporção (CARRAHER; SHLIEMANN, 1992; CARRAHER, CARRAHER; SHLIEMANN; RUIZ, 1986; TINOCO; LOPES, 1994; LOPES, 2008; SILVA; ALMOULOUD; 2008), poucos trabalhos estudaram a forma como essa regra é ensinada. Conforme verificaram Santos, Bay e 


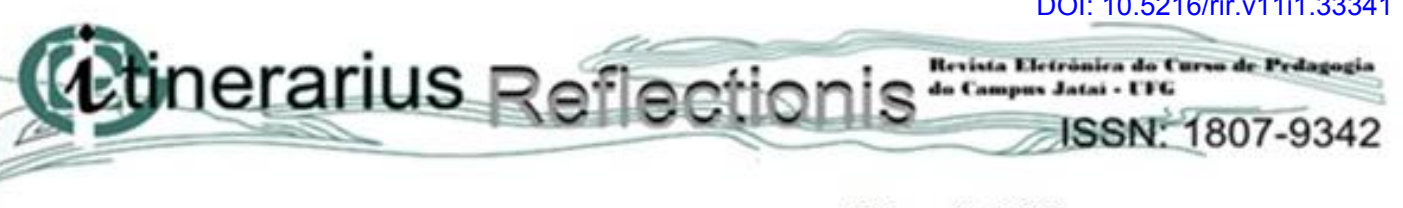

$$
\text { v.11, n. } 1,2015
$$

Souza (1997), a apresentação de uma só vez dos passos necessários descritos pela regra de três não aumentou o desempenho dos participantes na resolução dos exercícios. Esses resultados corroboram sugestões feitas por Albuquerque e Ferreira (2001) que verificaram existir uma relação inversa entre o tamanho da instrução e a probabilidade dela ser seguida. Portanto, seria interessante investigar o efeito da apresentação gradual das instruções descritas pela regra de três sobre a aquisição do conceito de proporção e na resolução de problemas sobre magnitude relativa entre frações. Um procedimento para esse fim pode ser a proposta de instrução programada feita por Skinner e ainda pouco explorada como alternativa ao ensino regular (SKINNER, 1972).

Instrução programada ou IP é uma denominação geral para todos os métodos de ensino cujo objetivo é programar a aprendizagem com o mínimo de erros possível. Um importante princípio desta proposta é que a aprendizagem será mais fácil se todos os prérequisitos (ou precorrentes) forem hierarquicamente ensinados. Em outras palavras, todas as relações comportamento-ambiente necessárias para aquisição de novas respostas devem ser ensinadas anteriormente, pois assim, diminui-se a possibilidade do aprendiz cometer erros. Segundo Skinner (1991), a instrução programada permite reforçar o comportamento do aprendiz imediatamente e substituir gradualmente os reforçadores artificiais pelos naturais à medida que o aluno avança dos estágios iniciais para os finais.

O presente experimento teve como objetivos investigar o efeito (1) do treino com instruções programadas sobre como resolver problemas fracionários, (2) do treino de relações condicionais entre estímulos pictóricos e numéricos e numéricos e numéricos segundo o paradigma proposto por Sidman e Taiby, (1982) e, (3) do treino conjunto entre instruções programadas e treino de relações condicionais sobre: a) formação classes de equivalência de estímulos; b) a expansão das classes de equivalência de estímulos formadas e a generalização para novas situações e c) a resolução de problemas fracionários do tipo lápis e papel.

\section{MÉTODO}

\subsection{Participantes}

Trinta alunos de final de sexta série do ensino fundamental, com idades entre $11 \mathrm{e}$ 13 anos, de ambos os sexos, que tiverem escores máximos iguais a 25\% de acertos em uma situação de teste denominada como avaliação inicial (descrita a seguir) e cujos responsáveis 


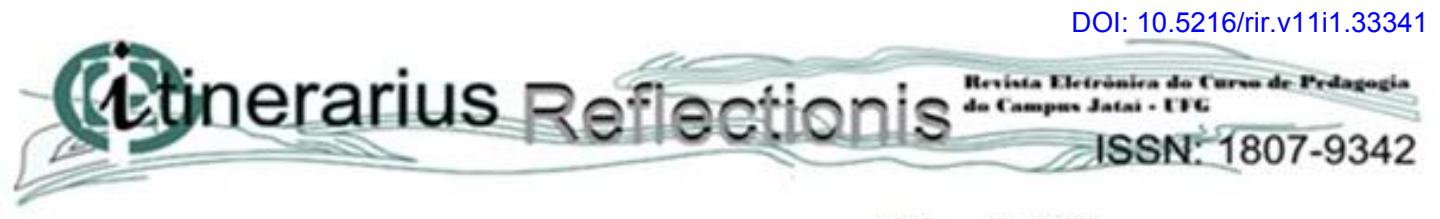

$$
\text { v.11, n. } 1,2015
$$

autorizaram a participação por meio do Termo de Autorização Livre e Esclarecido.

\subsection{Material}

Oito pastas do tipo classificador contendo folhas tamanho A4, no formato paisagem do Microsoft Word 2003. Cada folha apresentou quatro quadrados de $5 \mathrm{~cm}$ x $5 \mathrm{~cm}$, sendo um na parte central superior da folha e os três outros lado a lado na parte inferior. Os estímulos de amostras eram apresentados no quadrado superior e os de comparação nos inferiores.

Apostilas com instruções programadas sobre as operações necessárias para a resolução dos problemas fracionários e protocolos para o registro das respostas emitidas pelos participantes em cada condição de treino e teste programados.

\subsubsection{Estímulos}

Estímulos visuais na forma de frações numéricas e figuras que representavam as frações foram organizados em quatro conjuntos de três membros (Tabela 1).

Tabela 1- Estímulos apresentados nas condições de treinos e testes das relações condicionais

\begin{tabular}{|c|c|c|c|c|c|c|c|}
\hline \multicolumn{4}{|c|}{ Treinos e Testes de Equivalência } & \multicolumn{4}{|c|}{ Testes de Generalização } \\
\hline Conjunto & 1 & 2 & 3 & Conjunto & 1 & 2 & 3 \\
\hline A & & & & $\mathrm{E}$ & $1 / 2$ & $1 / 6$ & $1 / 7$ \\
\hline B & $1 / 3$ & $1 / 4$ & $1 / 5$ & $\mathrm{~F}$ & $2 / 4$ & $2 / 12$ & $2 / 14$ \\
\hline $\mathrm{C}$ & $2 / 6$ & $2 / 8$ & $2 / 10$ & G & $3 / 6$ & $3 / 18$ & $3 / 21$ \\
\hline $\mathrm{D}$ & $3 / 9$ & $3 / 12$ & $3 / 15$ & - & - & - & - \\
\hline
\end{tabular}

Fonte: Tabela construída pelos autores.

\subsection{Local}

O experimento ocorreu em uma sala de aula com aproximadamente 80 metros quadrados de uma escola da rede pública estadual de Goiânia. Na sala havia carteiras escolares individuais e lousa. A iluminação do ambiente era natural e artificial e a ventilação natural. 


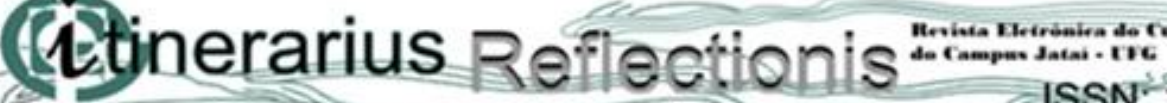

$$
\text { v.11, n. } 1,2015
$$

\subsection{Procedimento}

Foram organizados seis grupos de cinco participantes. Um grupo passou por treinos e testes de relações condicionais de acordo com o paradigma de equivalência proposto por Sidman e Tailby (1982) e foi denominado como grupo Equivalência (GEQ). Outro recebeu instruções programadas descrevendo gradualmente a regra de três no lugar dos treinos de relações condicionais e formou o grupo Instrução Programada (GIP) e outro recebeu instruções programadas mais treino de relações condicionais e formou o grupo Instrução Programada mais Equivalência (GIPEQ). Cada grupo experimental teve um de controle que fez apenas as avaliações Inicial e Final (descritas a seguir).

\subsubsection{Grupo Equivalência}

Os participantes foram expostos, em ordem, às seguintes condições de treinos e testes de relações condicionais: Avaliação inicial, treino $\mathrm{AB}$, treino $\mathrm{BC}$, treino $\mathrm{AB} / \mathrm{BC}$, teste de simetria (BA/CB), teste de transitividade/equivalência (AC/CA), teste de generalização 1, treino $\mathrm{AD}$, teste $\mathrm{D}-(\mathrm{ABC})$, teste de generalização 2 e avaliação final. A Tabela 2 descreve resumidamente as condições programadas para cada um dos grupos.

Tabela 2 - Organização das condições programadas, relações testadas e treinadas

\begin{tabular}{|c|c|c|c|c|c|}
\hline Condição & Relações Apresentadas & \multicolumn{4}{|c|}{ Grupos } \\
\hline & & GEQ & GIP & GIPEQ & Controles \\
\hline Avaliação Inicial & Ver no texto & Sim & Sim & Sim & Sim \\
\hline $\begin{array}{l}\text { Treino de } \\
\text { conhecimentos } \\
\text { básicos }\end{array}$ & Ver no texto & Sim & Sim & Sim & Não \\
\hline Treino da tarefa & Ver no Texto & Sim & Sim & Sim & Não \\
\hline Treino AB & $\mathrm{A} 1 \mathrm{~B} 1, \mathrm{~A} 2 \mathrm{~B} 2$ e $\mathrm{A} 3 \mathrm{~B} 3$ & Sim & Não & Sim & Não \\
\hline $\begin{array}{l}\text { Treino Instrução } \\
\text { Programada AB }\end{array}$ & Idem Treino AB & Não & Sim & Sim & Não \\
\hline Treino BC & $\mathrm{B} 1 \mathrm{C} 1, \mathrm{~B} 2 \mathrm{C} 2$ e B3C3 & Sim & Não & Sim & Não \\
\hline $\begin{array}{l}\text { Treino Instrução } \\
\text { Programada BC }\end{array}$ & Idem Treino BC & Não & Sim & Sim & Não \\
\hline Treino Misto & $\begin{array}{l}\text { A1B1, A2B2, A3B3, B1C1, } \\
\text { B2C2 е B3C3 }\end{array}$ & Sim & Não & Sim & Não \\
\hline Teste de Simetria & $\begin{array}{l}\text { B1A1, B2A2,B3A3, C1B1, } \\
\text { C2B2 е C3B3 }\end{array}$ & Sim & Sim & Sim & Não \\
\hline $\begin{array}{l}\text { Treino Instrução } \\
\text { Programada BA e CB }\end{array}$ & Idem Treino de Simetria & Não & Sim & Sim & Não \\
\hline Treino de simetria & B1A1, B2A2,B3A3, C1B1, & Sim & Sim & Sim & Não \\
\hline
\end{tabular}




\section{(2.tinerarius Refilectionis $=$ \\ ISSN: $1807-9342$}

v.11, n. 1,2015

\begin{tabular}{|c|c|c|c|c|c|}
\hline & C2B2 e C3B3 & & & & \\
\hline $\begin{array}{l}\text { Teste de } \\
\text { Transitividade e } \\
\text { equivalência }\end{array}$ & $\begin{array}{l}\mathrm{A} 1 \mathrm{C} 1, \mathrm{~A} 2 \mathrm{C} 2, \mathrm{~A} 3 \mathrm{C} 3, \mathrm{C} 1 \mathrm{~A} 1, \\
\mathrm{C} 2 \mathrm{~A} 2 \text { e } \mathrm{C} 3 \mathrm{~A} 3\end{array}$ & Sim & Sim & Sim & Não \\
\hline $\begin{array}{l}\text { Treino Instrução } \\
\text { Programada AC e CA }\end{array}$ & $\begin{array}{l}\text { Idem treino de Transitividade } \\
\text { e equivalência }\end{array}$ & Não & Sim & Sim & Não \\
\hline $\begin{array}{l}\text { Treino de } \\
\text { Transitividade e } \\
\text { equivalência }\end{array}$ & $\begin{array}{l}\mathrm{A} 1 \mathrm{C} 1, \mathrm{~A} 2 \mathrm{C} 2, \mathrm{~A} 3 \mathrm{C} 3, \mathrm{C} 1 \mathrm{~A} 1, \\
\mathrm{C} 2 \mathrm{~A} 2 \text { e } \mathrm{C} 3 \mathrm{~A} 3\end{array}$ & Sim & Não & Sim & Não \\
\hline $\begin{array}{l}\text { Teste de } \\
\text { Generalização } 1\end{array}$ & Ver no texto & Sim & Sim & Sim & Não \\
\hline Treino AD & $\mathrm{A} 1 \mathrm{D} 1, \mathrm{~A} 2 \mathrm{D} 2$ e A3D3 & Sim & Não & Sim & Não \\
\hline $\begin{array}{l}\text { Treino Instrução } \\
\text { Programada AD }\end{array}$ & Idem Treino AD & Não & Sim & & Não \\
\hline $\begin{array}{l}\text { Teste de Expansão das } \\
\text { classes D-(ABC) }\end{array}$ & $\begin{array}{l}\text { D1A1, D2A2, D3A3, D1B1, } \\
\text { D2B2, D3B3, B1D1, B2D2, } \\
\text { B3D3, C1D1, C2D2, C3D3, } \\
\text { D1C1, D2C2 e D3C3 }\end{array}$ & Sim & Sim & Sim & Não \\
\hline $\begin{array}{l}\text { Treino de Expansão } \\
\text { das classes D-(ABC) }\end{array}$ & $\begin{array}{l}\text { Idem relações do teste de } \\
\text { expansão }\end{array}$ & Sim & Não & Sim & Não \\
\hline $\begin{array}{l}\text { Treino Instrução } \\
\text { Programada D-(ABC) }\end{array}$ & $\begin{array}{l}\text { Idem relações do teste de } \\
\text { expansão }\end{array}$ & Não & Sim & Sim & Não \\
\hline $\begin{array}{l}\text { Teste de } \\
\text { Generalização } 2\end{array}$ & Ver no texto & Sim & Sim & Sim & Não \\
\hline Avaliação Final & Ver no texto & Sim & Sim & Sim & Sim \\
\hline
\end{tabular}

\subsubsection{Avaliação Inicial}

A avaliação inicial consistiu na aplicação coletiva de uma prova contendo 30 problemas fracionários, sendo que 10 problemas apresentaram relações absolutas entre as frações e 20 relações relativa (Detalhes da prova podem ser encontrados em Santos, 1999).

\subsubsection{Treino da Tarefa}

Todos os participantes receberam inicialmente treino em tarefa de pareamento com modelo com estímulos não fracionários. Por exemplo, uma bolinha como modelo e os numerais 1, 2 e 3 como comparação. O treino apresentou dois blocos com nove tentativas. Os estímulos foram apresentados nas pastas descritas no material. Os estímulos de modelo apareceram no quadrado superior e as três comparações nos quadrados inferiores. Os acertos foram consequenciados com a vocalização "certo" e os erros "errado". Na presença de erros um procedimento de correção vigorou até que o participante acertasse. As instruções orais 


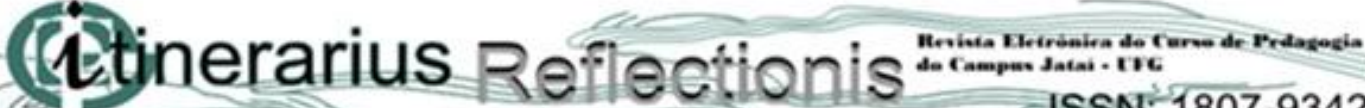 D.

$$
\text { v.11, n. } 1,2015
$$

para o treino inicial da tarefa foram as seguintes:

"Sua tarefa é simples. Você deverá em primeiro lugar tocar no quadrado da parte de cima da folha. Em seguida você deverá tocar uma das figuras laterais. Eu lhe direi se sua escolha foi certa ou errada. Tente acertar o máximo possível. Você entendeu?".

\subsubsection{Treinos AB, BC e AD}

Cada condição de treino foi precedida por um bloco com nove tentativas das relações que seriam ensinadas para as quais não houve nenhuma consequência programada e que foi denominado como pré-teste. Apenas quem teve menos que $100 \%$ de acertos no pré-teste fez o treino da condição. Após o treino, o bloco foi novamente aplicado e denominado como pósteste. As seguintes instruções orais antecederam os pré- e pós-testes:

"Sua tarefa continua sendo simples. Primeiramente, toque o quadrado que está na parte de cima da folha e em seguida toque uma das frações abaixo. Não será dito a você se sua escolha foi certa ou errada. Portanto, você poderá estar errando ou acertando em suas escolhas. Tente acertar o máximo que você puder. Entendeu?"

Nas condições dos treinos $\mathrm{AB}$ e $\mathrm{AD}$ ensinaram-se relações entre estímulos pictóricos como amostra (conjunto A) e numéricos (conjuntos B e D) como comparações. No treino BC tanto os estímulos de amostra (B) quanto os de comparação (C) foram numéricos. A definição alfanumérica (A1B1, B1C1, A1D1, etc) determinou quais relações foram consideradas certas ou erradas (ver Tabela 1). A apresentação dos estímulos foi igual ao do treino da tarefa, sendo que as comparações corretas apareceram em posições diferentes a cada tentativa. Cada relação foi apresentada três vezes formando um bloco de treino com nove tentativas. Cada condição de treino apresentou alternadamente dois blocos que diferiram apenas quanto à ordem de apresentação das tentativas. Para esses treinos foram dadas as seguintes instruções orais:

"Sua tarefa continua sendo simples. Tudo o que você tem que fazer é tocar na figura da parte de cima da folha e em seguida, tocar uma das frações abaixo. No entanto, agora será dito a você se sua escolha foi certa ou errada. Tente acertar o máximo possível. Você entendeu?". 


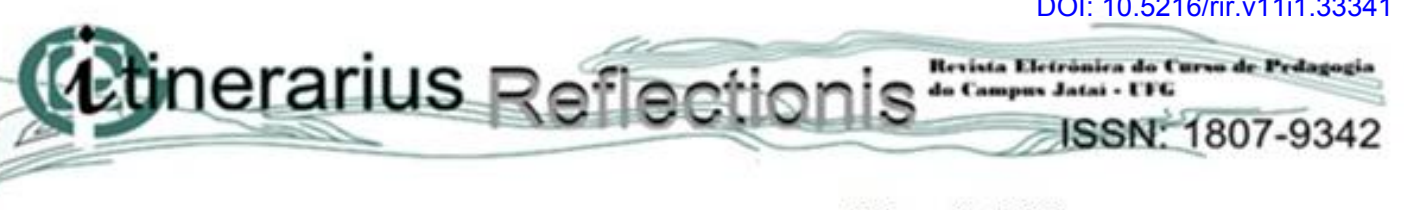

$$
\text { v.11, n. } 1,2015
$$

\subsubsection{Treino $\mathrm{AB} / \mathrm{BC}$}

No treino $\mathrm{AB} / \mathrm{BC}$ foram aplicados dois blocos com 12 tentativas. Cada bloco foi formado por seis tentativas com relações $\mathrm{AB}$ e seis $\mathrm{BC}$. Os blocos diferiram apenas quanto a ordem de apresentação das tentativas e apenas três delas em cada bloco foram consequenciadas.

"A sua tarefa é igual à anterior. Tudo que você tem que fazer é tocar na figura da parte de cima da folha e em seguida tocar uma das frações laterais. Apenas algumas vezes será dito a você se sua escolha foi certa ou errada. Quando nada for dito você pode ter acertado ou errado. Entendeu?".

O critério adotado para o encerramento de todas as condições de treino foi o acerto de $100 \%$ das tentativas a partir da exposição ao segundo bloco.

\subsubsection{Condições de testes}

Os testes avaliaram a emergência das relações BA/CB (simetria) e AC/CA (transitividade/equivalência), expansão D-(ABC) e generalização. Cada teste foi programado separadamente e consistiu na apresentação alternada de dois blocos de 12 tentativas cada. No teste de simetria três tentativas foram das relações BA, três das relações $\mathrm{CB}$ e seis dos treinos $\mathrm{AB}$ e BC (três de cada relação). De forma semelhante, no teste de transitividade/equivalência, três foram da relação AC, três da CA e seis das relações AB e BC. Os dois blocos dessas duas condições diferiram apenas quanto a ordem de apresentação das tentativas. Apenas quatro, das seis tentativas de treino de cada bloco foram consequenciadas. $O$ teste $\mathrm{D}-(\mathrm{ABC})$ avaliou a ocorrência das seguintes relações: DA, DB, BD, DC e CD. Apresentaram-se alternadamente dois blocos formados por três tentativas de cada relação (variando a posição da comparação correta) e mais cinco tentativas de linha de base que foram as únicas consequenciadas. A ordem das tentativas variou em cada bloco. Participantes que não demonstraram o critério nessas condições de testes fizeram o treino direto dessas relações. Nos treinos, todas as tentativas apresentadas nos testes foram consequenciadas. As instruções foram as mesmas das condições do treino $\mathrm{AB} / \mathrm{BC}$.

Os testes se encerraram mediante dois critérios. Primeiro, com 100\% de acertos a partir do segundo bloco de tentativas. Segundo, caso o participante cometesse erros no primeiro bloco faria mais blocos até demonstrar $100 \%$ de acerto. Se o desempenho diminuísse 
de um bloco para outro o teste foi suspenso e as relações treinadas diretamente segundo critérios dos treinos anteriores.

O teste de generalização 1 avaliou as relações E1F1, E2F2, E3F3, F1E1, F2E2 e F3E3. Apresentou-se um bloco com três tentativas de cada relação (variando-se a posição da comparação correta) e mais quatro de linha de base que foram consequenciadas. No teste de generalização 2 foram avaliadas as relações E1G1, E2G2, E3G3, G1E1, G2E2, G3E3, F1G1, F2G2, F3G3. Apresentou-se um bloco com três tentativas de cada relação (variando-se a posição da comparação correta) e seis de linha de base que foram consequenciadas. As instruções foram as mesmas da condição de treino AB/BC. Após o teste de generalização 2 os participantes refizeram os problemas aplicados na avaliação inicial que agora foi chamada de avaliação final.

\subsubsection{Grupo Instrução Programada}

O grupo Instrução Programada (GIP) passou pelas mesmas condições e critérios do grupo equivalência (GEQ). Contudo, os participantes que não demonstraram o critério nos pré-testes das condições $\mathrm{AB}, \mathrm{BC}, \mathrm{AD}$ e nos testes de simetria e transitividade/equivalência, passaram pelo treino dessas relações por meio de instruções programadas. Esses treinos consistiram na aplicação de exercícios graduais sobre os passos e as operações de multiplicação e divisão necessárias para a resolução dos problemas fracionários apresentados em cada um dessas condições. Cada treino foi apresentado em apostilas com um exercício de cada relação testada. Por exemplo, se a relação apresentasse o estímulo pictórico correspondente a 1/3 como modelo e as frações 1/3, 1/4 e 1/5 como comparação (relação AB), o participante foi instruído a contar o número de partes em que a figura foi dividida e colocar o resultado no lugar indicado para o denominador e contar o número de partes que estava sombreada na figura e colocar no lugar indicado para o numerador. Se o estímulo de modelo fosse a fração numérica 1/3 e os de comparação fossem as frações 2/6, 2/8 e 3/9 (relação BC), o participante foi instruído, passo a passo, a multiplicar o número um por dois e colocar o resultado no lugar indicado para o numerador e multiplicar o número três por dois e colocar o resultado no lugar indicado para o denominador. Problemas envolvendo operações de divisão (relação CB, por exemplo) foram instruídos de forma semelhante.

Os participantes tiveram tempo livre para resolver os exercícios na presença do 


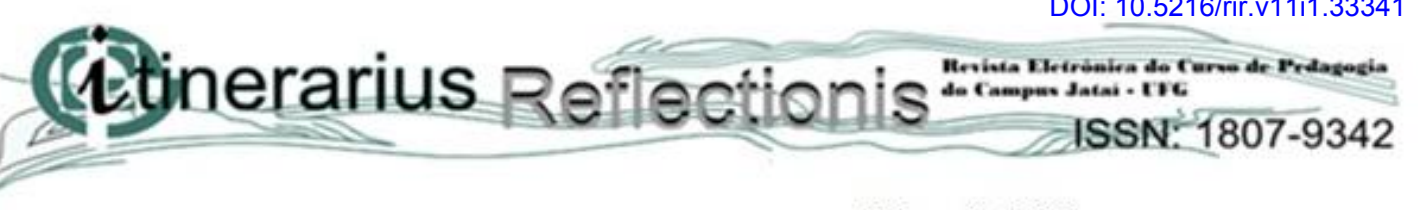

$$
\text { v.11, n. } 1,2015
$$

experimentador, o qual corrigia os problemas imediatamente. Após demonstrarem $100 \%$ de acertos os participantes fizeram o pós-teste. As instruções orais dadas aos participantes nessas condições foram as seguintes:

"Você irá agora resolver alguns exercícios sobre os problemas que você acabou de fazer. Os exercícios estão nessa apostila e serão resolvidos a lápis. Leia as instruções com bastante atenção. Quando terminar me avise e eu lhe direi quais foram resolvidos corretamente e quais ficaram incorretos. Se você errar algum fará todo exercício novamente. Você Entendeu?".

\subsubsection{Grupo Instrução Programada mais Equivalência}

O grupo Instrução Programada mais Equivalência (GIPEQ) passou pelas mesmas condições do grupo Instrução Programada mais as do grupo Equivalência. Nessa condição os participantes que não demonstram $100 \%$ de acerto nos pré-testes das condições $\mathrm{AB}, \mathrm{BC}$ e $\mathrm{AD}$ e nos testes de simetria e transitividade/equivalência, resolveram primeiro os exercícios na forma de instrução programada e imediatamente após demonstrarem o critério passaram pelo treino de relações condicionais segundo o paradigma proposto por Sidman e Tailby (1982). Os critérios e instruções foram os mesmos dos grupos GEQ e GIP.

\section{RESULTADOS}

O delineamento programou avaliações dos desempenhos dos participantes ao longo de várias condições experimentais, o que enfatiza o interesse no dado individual. Contudo, dados foram também analisados comparando-se os desempenhos dos grupos, o que permitiu investigar o efeito isolado do treino de relações condicionais e do treino com instruções programadas, bem como a interação de ambos sobre a formação de classes de equivalência e a expansão das classes formadas e o desempenho generalizado.

A Tabela 3 apresenta a porcentagem de acertos para cada participante dos três grupos experimentais e de controles nas condições de avaliação inicial e final. Os resultados mostraram porcentagens médias de acertos maiores em problemas cujas frações foram expressas de forma absoluta em todos os grupos. $\mathrm{Na}$ avaliação inicial dos grupos experimentais, a maior porcentagem media de acertos ocorreu para o grupo GEQ (84\%). Em seguida, vieram os grupos GIPEQ e GIP com $78 \%$ e $64 \%$, respectivamente. Nos grupos de 


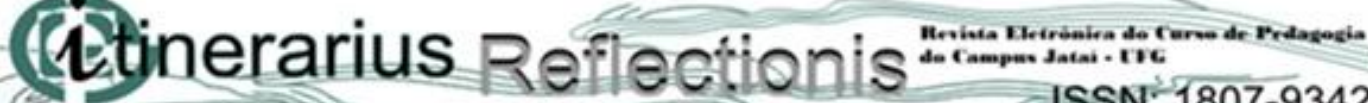

\section{v.11, n. 1,2015}

controle os valores oscilaram entre $72 \%$ e $94 \%$. Por outro lado, quando as frações foram expressas de forma relativa os escores diminuíram para todos os grupos. Nos experimentais, as porcentagens medias de acertos foram, em sequência, $9 \%, 4 \%$ e $0 \%$ para os grupos GIP, GEQ e GIPEC e nos controles os valores oscilaram entre $12 \%$ e $20 \%$. Na avaliação final, houve aumento na porcentagem média de acertos para todos os grupos experimentais, tanto quando as frações foram expressas de forma absoluta quanto relativa. O maior ganho na porcentagem média de acertos para frações expressas de forma relativa ocorreu para o grupo GIPEQ, onde os índices passaram de 0\% para 69\%. O segundo maior ganho foi para o GEQ onde os valores subiram de $4 \%$ para $53 \%$ e, por último para GIP, cuja média passou de $9 \%$ para $48 \%$. Entre os grupos de controle, apenas no GIPEQ houve aumento na porcentagem de acertos da avaliação inicial para a final quando as frações foram expressas de forma absoluta (passou de $72 \%$ para 74\%). Quando as frações foram expressas de forma relativa houve aumentos nas porcentagens médias de acertos do GEQ (12\% para 34\%) e do GIPEQ (20\% para $25 \%)$.

Tabela 3 - Porcentagem (individual e média) de acertos nas frações expressas de forma absoluta e relativa.

\begin{tabular}{|c|c|c|c|c|c|c|c|c|c|}
\hline \multicolumn{10}{|c|}{ GEQ } \\
\hline \multicolumn{5}{|c|}{ Grupo Experimental } & \multicolumn{5}{|c|}{ Grupo Controle } \\
\hline \multicolumn{3}{|c|}{ Absolutas } & \multicolumn{2}{|c|}{ Relativas } & & \multicolumn{2}{|c|}{ Absolutas } & \multicolumn{2}{|c|}{ Relativas } \\
\hline Participante & A.I & A.F & A.I & A.F & Participante & A.I & A.F & A.I & A.F \\
\hline $\mathrm{P} 1$ & 50 & 80 & 0 & 30 & $\mathrm{P} 21$ & 90 & 100 & 30 & 35 \\
\hline $\mathrm{P} 2$ & 100 & 90 & 5 & 20 & $\mathrm{P} 22$ & 80 & 70 & 0 & 5 \\
\hline P3 & 90 & 90 & 5 & 50 & $\mathrm{P} 23$ & 90 & 100 & 5 & 85 \\
\hline P4 & 100 & 90 & 5 & 70 & P24 & 100 & 90 & 15 & 35 \\
\hline P5 & 80 & 80 & 5 & 95 & P25 & 100 & 100 & 10 & 10 \\
\hline $\bar{X}$ & 84 & 86 & 4 & 53 & $\bar{X}$ & 92 & 92 & 12 & 34 \\
\hline \multicolumn{10}{|c|}{ GIP } \\
\hline \multicolumn{5}{|c|}{ Grupo Experimental } & \multicolumn{5}{|c|}{ Grupo Controle } \\
\hline & \multicolumn{2}{|c|}{ Absolutas } & \multicolumn{2}{|c|}{ Relativas } & & \multicolumn{2}{|c|}{ Absolutas } & \multicolumn{2}{|c|}{ Relativas } \\
\hline Participante & A.I & A.F & A.I & A.F & Participante & A.I & A.F & A.I & A.F \\
\hline P6 & 40 & 90 & 0 & 10 & P16 & 90 & 100 & 10 & 0 \\
\hline P7 & 70 & 100 & 0 & 75 & P17 & 100 & 90 & 10 & 5 \\
\hline P8 & 100 & 100 & 25 & 65 & P18 & 100 & 50 & 65 & 15 \\
\hline P9 & 70 & 90 & 0 & 55 & P19 & 80 & 70 & 15 & 15 \\
\hline $\mathrm{P} 10$ & 40 & 100 & 20 & 35 & $\mathrm{P} 20$ & 100 & 90 & 0 & 5 \\
\hline $\bar{X}$ & 64 & 96 & 9 & 48 & $\bar{X}$ & 94 & 80 & 20 & 8 \\
\hline \multicolumn{10}{|c|}{ GIPEQ } \\
\hline \multicolumn{5}{|c|}{ Grupo Experimental } & & \multicolumn{4}{|c|}{ Grupo Controle } \\
\hline \multicolumn{3}{|c|}{ Absolutas } & \multicolumn{2}{|c|}{ Relativas } & & \multicolumn{2}{|c|}{ Absolutas } & \multicolumn{2}{|c|}{ Relativas } \\
\hline
\end{tabular}




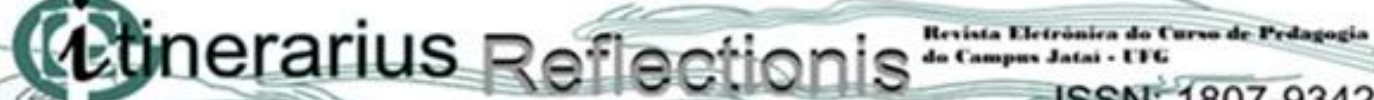 20

v.11, n. 1,2015

\begin{tabular}{cccccccccc}
\hline Participante & A.I & A.F & A.I & A.F & Participante & A.I & A.F & A.I & A.F \\
P11 & 70 & 100 & 0 & 75 & P26 & 60 & 100 & 20 & 30 \\
P12 & 90 & 100 & 0 & 60 & P27 & 90 & 80 & 10 & 20 \\
P13 & 50 & 80 & 0 & 60 & P28 & 80 & 80 & 20 & 30 \\
P14 & 80 & 90 & 0 & 70 & P29 & 60 & 50 & 30 & 10 \\
P15 & 100 & 90 & 0 & 80 & P30 & 70 & 60 & 20 & 35 \\
$\bar{X}$ & 78 & 92 & 0 & 69 & $\bar{X}$ & 72 & 74 & 20 & 25 \\
\hline \multicolumn{1}{c}{ Fonte: Tabela construída pelos autores. }
\end{tabular}

A Tabela 4 apresenta a porcentagem de acertos nos pré- e pós-testes das condições $\mathrm{AB}, \mathrm{BC}$ e $\mathrm{AD}$ e o número de blocos para demonstrar o critério na condição de treino $\mathrm{AB} / \mathrm{BC}$ para os participantes dos grupos GEQ, GIP e GIPEQ. Os resultados mostraram porcentagens médias de acertos de 100\% para os grupos GEQ e GIPEQ. Treze dos 15 participantes tiveram $100 \%$ de acertos no pré-teste da condição AB. Apenas P6 e P10 fizeram o treino direto dessas relações, mas demonstraram o critério no pós-teste. Nas condições de treino $\mathrm{BC}$ e $\mathrm{AD}$, as porcentagens médias de acertos nos pré-testes ficaram abaixo de $100 \%$ nos três grupos. Na condição BC, apenas oito participantes (P4, P5, P7, P8, P9, P10, P11 e P14) demonstraram $100 \%$ de acertos. Na condição $\mathrm{AD}$, apenas seis participantes (P1, P4, P5, P10, P14 e P15) tiveram $100 \%$ de acertos. Os demais participantes tiveram escores inferiores e passaram pelo treino direto das relações, mas todos tiveram $100 \%$ de acertos no pós-teste. Todos os participantes (exceto P9) tiveram 100\% de acertos na condição de treino AB/BC em no máximo dois blocos de tentativas.

Tabele 4 - Porcentagem de acertos nas condições de pré e pós-testes nas condições $\mathrm{AB}, \mathrm{BC}$, e $\mathrm{AD}$ e número de blocos de treino na condição $\mathrm{AB} / \mathrm{BC}$

\begin{tabular}{ccccccccc}
\hline \multicolumn{1}{c}{ AB } & \multicolumn{9}{c}{ BC } & AB/BC & \multicolumn{2}{c}{ AD } \\
\hline Grupo & Partic. & Pré-t. & Pós-t. & Pré-t. & Pós-t. & NB & Pré-t. & Pós-t. \\
& P1 & 100 & - & 89 & 100 & 2 & 100 & - \\
& P2 & 100 & - & 89 & 100 & 2 & 55 & 100 \\
GEQ & P3 & 100 & - & 55 & 100 & 2 & 89 & 100 \\
& P4 & 100 & - & 100 & - & 2 & 100 & - \\
& P5 & 100 & - & 100 & - & 2 & 100 & - \\
& $\bar{X}$ & 100 & 100 & 86,6 & 100 & 2 & 88,8 & 100 \\
& P6 & 22 & 100 & 33 & 100 & 2 & 88 & 100 \\
GIP & P7 & 100 & - & 100 & - & 2 & 55 & 100 \\
& P8 & 100 & - & 100 & - & 2 & 78 & 100 \\
& P9 & 100 & - & 100 & - & 3 & 66 & 100
\end{tabular}




\section{(4tinerarius Reflectionis $=$

$$
\text { v.11, n. } 1,2015
$$

\begin{tabular}{|c|c|c|c|c|c|c|c|c|}
\hline & P10 & 64 & 100 & 100 & - & 2 & 100 & - \\
\hline \multirow{7}{*}{ GIPEQ } & $\bar{X}$ & 77.2 & 100 & 86,6 & 100 & 2,2 & 77 & 100 \\
\hline & P11 & 100 & - & 100 & - & 2 & 44 & 100 \\
\hline & P12 & 100 & - & 33 & 100 & 2 & 66 & 100 \\
\hline & P13 & 100 & - & 44 & 100 & 2 & 44 & 100 \\
\hline & P14 & 100 & - & 100 & - & 2 & 100 & - \\
\hline & P15 & 100 & - & 33 & 100 & 2 & 100 & - \\
\hline & $\bar{X}$ & 100 & - & 62 & 100 & 2 & 71 & 100 \\
\hline
\end{tabular}

A Tabela 5 apresenta a porcentagem de acertos por bloco de tentativa nos testes das condições BA/CB (simetria), AC/CA (transitividade/equivalência) e expansão da rede D(ABC) para os participantes dos grupos, Equivalência (GEQ), Instrução Programada (GIP) e Instrução Programada mais Equivalência (GIPEQ). Na condição de teste BA/CB (simetria), todos os participantes, exceto P6 e P13, tiveram 100\% de acertos em dois blocos de tentativas. Estes dois participantes fizeram o treino direto das relações e demonstraram o critério em dois blocos. Na condição de teste AC/CA (Transitividade/equivalência), 10 dos 15 participantes (P3, P4, P5, P8, P9, P10, P11, P12 P14 E P15) demonstraram o critério em dois blocos de tentativas e outros dois, P6 e P7, em quatro e cinco blocos, respectivamente. Apenas três participantes (P1, P2 e P13) precisaram de treino direto dessas relações e demonstraram o critério em dois blocos de tentativas. No teste de expansão da rede $\mathrm{D}-(\mathrm{ABC})$, apenas dois participantes (P4 e P5) demonstraram 100\% de acertos, sendo que P5 precisou de dois blocos de tentativas e P4 treze (este participante obteve $87 \%$ de acertos do segundo ao décimo segundo blocos e por isso parte dos dados foram suprimidos). Os demais 13 participantes demonstraram escores decrescentes ao longo de até sete blocos de tentativas e passaram pelo treino direto dessas relações. Todos aprenderam as relações em oito blocos de tentativas, exceto P1 e P8 que precisaram de 13 e 21 blocos, respectivamente. (os dados dos treinos dessas três condições não aparecem nas tabelas).

Tabela 5 - Porcentagem de acertos por bloco de tentativa (B) nas condições de teste de BA/CB (simetria),

AC/CA (simetria), AC/CA (transitividade/equivalência) e Expansão da rede D-(ABC)

\begin{tabular}{lllllllllllllllll}
\hline & \multicolumn{1}{c}{ Simetria } & \multicolumn{1}{c}{ Transitividade/equivalência } & \multicolumn{1}{c}{ Expansão D-(ABC) } \\
\hline Grupo & Part & B1 & B2 & B1 & B2 & B3 & B4 & B5 & B1 & B2 & B3 & B4 & B5 & B6 & B7 & B13 \\
& P1 & 100 & 100 & 83 & 67 & - & - & - & 80 & 80 & 70 & - & - & - & - & - \\
& P2 & 100 & 100 & 83 & 67 & - & - & - & 47 & 67 & 60 & - & - & - & - & - \\
& P3 & 83 & 100 & 67 & 100 & - & - & - & 80 & 93 & 73 & - & - & - & - & -
\end{tabular}




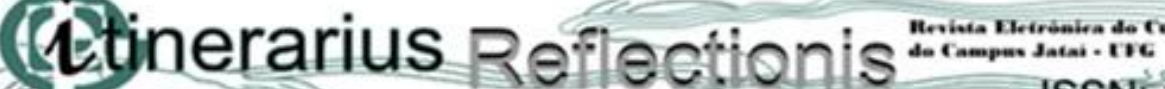 \\ ISSN: $1807-9342$}

$$
\text { v.11, n. } 1,2015
$$

\begin{tabular}{|c|c|c|c|c|c|c|c|c|c|c|c|c|c|c|c|c|}
\hline \multirow[t]{6}{*}{ GEQ } & P4 & 100 & 100 & 100 & 100 & - & - & - & 73 & 87 & 87 & 87 & 87 & 87 & 87 & 100 \\
\hline & P5 & 100 & 100 & 100 & 100 & - & - & - & 93 & 100 & - & - & - & - & - & - \\
\hline & $\bar{X}$ & 97 & 100 & 87 & 87 & - & - & - & 75 & 85 & 72 & - & - & - & - & - \\
\hline & P6 & 83 & 66 & 50 & 67 & 67 & 67 & 100 & 73 & 80 & 80 & 66 & - & - & - & - \\
\hline & P7 & 100 & 100 & 83 & 83 & 83 & 100 & - & 60 & 73 & 73 & 73 & 80 & 93 & 80 & - \\
\hline & P8 & 100 & 100 & 100 & 100 & - & - & - & 80 & 80 & 80 & 73 & - & - & - & - \\
\hline \multirow[t]{5}{*}{ GIP } & P9 & 100 & 100 & 100 & 100 & - & - & - & 80 & 80 & 60 & - & - & - & - & - \\
\hline & P10 & 100 & 100 & 89 & 100 & - & - & - & 80 & 80 & 80 & 80 & 86 & 80 & - & - \\
\hline & $\bar{X}$ & 97 & 100 & 84 & 90 & 75 & 83 & - & 75 & 79 & 75 & 73 & 83 & - & - & - \\
\hline & P11 & 100 & 100 & 66 & 100 & - & - & - & 60 & 73 & 40 & - & - & - & - & - \\
\hline & $\mathrm{P} 12$ & 83 & 100 & 83 & 100 & - & - & - & 73 & 60 & - & - & - & - & - & - \\
\hline \multirow[t]{4}{*}{ GIPEQ } & P13 & 66 & 66 & 66 & 33 & - & - & - & 47 & 27 & - & - & - & - & - & - \\
\hline & P14 & 100 & 100 & 66 & 100 & - & - & - & 60 & 53 & - & - & - & - & - & - \\
\hline & $\mathrm{P} 15$ & 100 & 100 & 100 & 100 & - & - & - & 47 & 47 & 40 & & - & - & - & - \\
\hline & $\bar{X}$ & 90 & 93 & 77 & 87 & - & - & - & 57 & 52 & 40 & - & - & - & - & - \\
\hline
\end{tabular}

Fonte: Tabela construída pelos autores.

A Tabela 6 apresenta os resultados dos testes de generalização 1 e 2 para os participantes dos grupos Equivalência (GEQ), Instrução Programada (GIP) e Equivalência mais Instrução Programada (GIPQ). As porcentagens médias de acertos foram maiores no teste de generalização 1 do que no teste 2 . No teste 1 a maior média ocorreu para o GIP (96.6\%), sendo que os participantes P2, P3, P4 e P5 acertaram todas as tentativas. O grupo GEQ teve média de $88.4 \%$ e três participantes, P6, P9 e P10, tiveram $100 \%$ de acertos. No GIPEC a média foi de $76.2 \%$ e apenas um participante (P11) teve $100 \%$ de acertos. No teste 2 a porcentagem média de acertos oscilou entre $56.4 \%$ e $64,4 \%$. Apenas dois participantes (P5 e P11) acertaram todas as tentativas. Os demais participantes tiveram porcentagens de acertos variando entre $36 \%$ e $78 \%$.

Tabela 6 - Porcentagem de acertos e média nas condições de teste de generalização 1 (TG1) e teste de generalização 2 (TG2)

\begin{tabular}{cccccccccccc}
\hline Cond. & Partic. & TG1 & TG2 & Cond. & Partic. & TG1 & TG2 & Cond. & Part. & TG1 & TG2 \\
\hline \multirow{6}{*}{ GEQ } & P1 & 100 & 59 & & P11 & 100 & 75 & & P6 & 83 & 36 \\
& P2 & 67 & 71 & & P12 & 66 & 58 & & P7 & 100 & 64 \\
& P3 & 75 & 39 & GIPEQ & P13 & 58 & 54 & GIP & P8 & 100 & 67 \\
& P4 & 100 & 46 & & P14 & 66 & 63 & & P9 & 100 & 37 \\
& P5 & 100 & 100 & & P15 & 91 & 72 & & P10 & 100 & 78 \\
& $\bar{X}$ & 88.4 & 63 & & $\bar{X}$ & 76.2 & 64.4 & & $\bar{X}$ & 96.6 & 56.4 \\
\hline \multicolumn{7}{c}{ Fonte: Tabela construída pelos autores. }
\end{tabular}




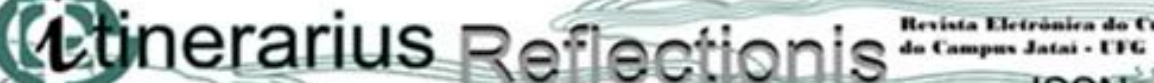 \\ ISSN: 1807-9342}

\section{v.11, n. 1,2015}

A Tabela 7 apresenta a porcentagem de acertos e a de erros ocorridos em cada comparação incorreta de cada relação no teste de expansão da rede para os grupos Instrução Programada (GIP), Equivalência (GEQ) e Instrução Programada mais Equivalência (GIPEQ). Em todas as relações testadas nos três grupos experimentais a porcentagem de acertos foi inferior a $100 \%$. De forma geral, os maiores índices ocorreram para os grupos GIP e GEQ, em especial nas relações três relações BD e DB, nas quais as porcentagens de acertos variaram entre $71 \%$ e $93 \%$. Apenas na relação D1A1 os escores foram iguais ou maiores que $75 \%$ nos três grupos. Os menores índices de acertos ocorreram no GIPEQ, cujas porcentagens de acertos oscilaram entre $40 \%$ e $67 \%$ (exceto em D1A1).

Tabela 7 - Porcentagem de acertos (PA) e de erros em cada relação testada na condição de teste de expansão das classes D-(ABC)

\begin{tabular}{|c|c|c|c|c|c|c|c|c|c|}
\hline \multirow{4}{*}{$\frac{\text { Relação }}{\text { D1A1 }}$} & \multicolumn{3}{|c|}{ GEQ } & \multicolumn{3}{|c|}{ GIP } & \multicolumn{3}{|c|}{ GIPEQ } \\
\hline & \multicolumn{3}{|c|}{$\begin{array}{l}\text { Erros } \\
\text { Iternati }\end{array}$} & PA & \multicolumn{2}{|c|}{$\begin{array}{c}\text { Erros } \\
\text { Alternativas }\end{array}$} & \multicolumn{3}{|c|}{$\begin{array}{l}\text { Erros } \\
\text { Alternativas }\end{array}$} \\
\hline & & $\mathrm{A} 2(1 / 4 \mathrm{~F})$ & $\mathrm{A} 3(1 / 5 \mathrm{~F})$ & & $\mathrm{A} 2(1 / 4 \mathrm{~F})$ & $\mathrm{A} 3(1 / 5 \mathrm{~F})$ & & $\mathrm{A} 2(1 / 4 \mathrm{~F})$ & $\mathrm{A} 3(1 / 5 \mathrm{~F})$ \\
\hline & 96 & 4 & 0 & 86 & 14 & 0 & 75 & 25 & 0 \\
\hline D2A2 & & $\mathrm{A} 1(1 / 3 \mathrm{~F})$ & $\mathrm{A} 3(1 / 5 \mathrm{~F})$ & & $\mathrm{A} 1(1 / 3 \mathrm{~F})$ & $\mathrm{A} 3(1 / 5 \mathrm{~F})$ & & $\mathrm{A} 1(1 / 3 \mathrm{~F})$ & $\mathrm{A} 3(1 / 5 \mathrm{~F})$ \\
\hline$(3 / 12-1 / 4 \mathrm{~F})$ & 83 & 17 & 0 & 57 & 36 & 7 & 47 & 6 & 47 \\
\hline D3A3 & & $\mathrm{A} 1(1 / 3 \mathrm{~F})$ & $\mathrm{A} 2(1 / 4 \mathrm{~F})$ & & $\mathrm{A} 1(1 / 3 \mathrm{~F})$ & $\mathrm{A} 2(1 / 4 \mathrm{~F})$ & & $\mathrm{A} 1(1 / 3 \mathrm{~F})$ & $\mathrm{A} 2(1 / 4 \mathrm{~F})$ \\
\hline$(3 / 15-1 / 5 F)$ & - & - & - & - & - & - & - & - & - \\
\hline B1D1 & & D2(3/12) & D3(3/15) & & D2(3/12) & D3(3/15) & & D2(3/12) & D3(3/15) \\
\hline$(1 / 3-3 / 9)$ & 92 & 8 & 0 & 86 & 14 & 0 & 67 & 26 & 7 \\
\hline B2D2 & & $\mathrm{D} 1(3 / 9)$ & D3(3/15) & & $\mathrm{D} 1(3 / 9)$ & D3(3/15) & & $\mathrm{D} 1(3 / 9)$ & D3(3/15) \\
\hline$(1 / 4-3 / 12)$ & 83 & 17 & 0 & 71 & 14 & 14 & 60 & 8 & 32 \\
\hline B3D3 & & D1(3/9) & D2(3/12) & & D1(3/9) & D2(3/12) & & D1(3/9) & D2(3/12) \\
\hline$(1 / 5-3 / 15)$ & 83 & 8 & 8 & 86 & 7 & 7 & 53 & 13 & 33 \\
\hline D1B1 & & B2(1/4) & $\mathrm{B} 3(1 / 5)$ & & B2(1/4) & B3(1/5) & & B2(1/4) & B3(1/5) \\
\hline$(3 / 9-1 / 3)$ & 83 & 0 & 17 & 86 & 14 & 0 & 53 & 38 & 9 \\
\hline D2B2 & & B1(1/3) & $\mathrm{B} 3(1 / 5)$ & & B1(1/3) & B3(1/5) & & B1(1/3) & B3(1/5) \\
\hline$(3 / 12-1 / 4)$ & 83 & 8 & 8 & 71 & 21 & 7 & 53 & 47 & 0 \\
\hline D3B3 & & $\mathrm{B} 1(1 / 3)$ & B2(1/4) & & $\mathrm{B} 1(1 / 3)$ & B2(1/4) & & $\mathrm{B} 1(1 / 3)$ & B2(1/4) \\
\hline$(3 / 15-1 / 5)$ & 92 & 0 & 8 & 93 & 7 & 0 & 43 & 14 & 43 \\
\hline C1D1 & & D2(3/12) & D3(3/15) & & D2(3/12) & D3(3/15) & & $\mathrm{D} 2(3 / 12)$ & D3(3/15) \\
\hline$(2 / 6-3 / 9)$ & 8 & 92 & 0 & 71 & 29 & 0 & 50 & 50 & 0 \\
\hline $\mathrm{C} 2 \mathrm{D} 2$ & & $\mathrm{D} 1(3 / 9)$ & D3(3/15) & & $\mathrm{D} 1(3 / 9)$ & D3(3/15) & & $\mathrm{D} 1(3 / 9)$ & D3(3/15) \\
\hline$(2 / 8-3 / 12)$ & 58 & 0 & 42 & 71 & 14 & 14 & 50 & 21 & 29 \\
\hline C3D3 & & $\mathrm{D} 1(3 / 9)$ & $\mathrm{D} 2(3 / 12)$ & & $\mathrm{D} 1(3 / 9)$ & $\mathrm{D} 2(3 / 12)$ & & $\mathrm{D} 1(3 / 9)$ & $\mathrm{D} 2(3 / 12)$ \\
\hline$(2 / 10-3 / 15)$ & 75 & 17 & 8 & 50 & 21 & 29 & 40 & 40 & 20 \\
\hline $\mathrm{D} 1 \mathrm{C} 1$ & & $\mathrm{C} 2(2 / 8)$ & C3(2/10) & & $\mathrm{C} 2(2 / 8)$ & $\mathrm{C} 3(2 / 10)$ & & $\mathrm{C} 2(2 / 8)$ & C3(2/10) \\
\hline$(3 / 9-2 / 6)$ & 29 & 0 & 71 & 79 & 14 & 7 & 50 & 10 & 40 \\
\hline
\end{tabular}




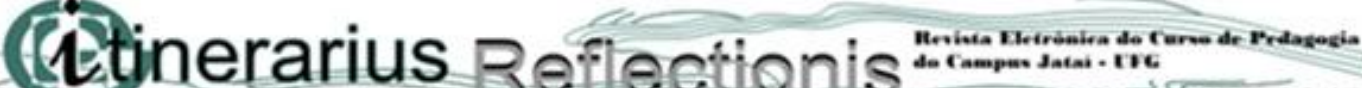 Des}

\begin{tabular}{cccccccccc}
\multicolumn{10}{c}{$\boldsymbol{v} \cdot 11, \boldsymbol{n . 1} \mathbf{1} 2015$} \\
$\mathrm{D} 2 \mathrm{C} 2$ & & $\mathrm{C} 1(2 / 6)$ & $\mathrm{C} 3(2 / 10)$ & & $\mathrm{C} 1(2 / 6)$ & $\mathrm{C} 3(2 / 10)$ & & $\mathrm{C} 1(2 / 6)$ & $\mathrm{C} 3(2 / 10)$ \\
$(3 / 12-2 / 8)$ & 0 & 100 & 0 & 50 & 38 & 14 & 40 & 53 & 6 \\
$\mathrm{D} 3 \mathrm{C} 3$ & & $\mathrm{C} 1(2 / 6)$ & $\mathrm{C} 2(2 / 8)$ & & $\mathrm{C} 1(2 / 6)$ & $\mathrm{C} 3(2 / 10)$ & & $\mathrm{C} 1(2 / 6)$ & $\mathrm{C} 3(2 / 10)$ \\
$(3 / 15-2 / 10)$ & 59 & 23 & 18 & 57 & 29 & 14 & 60 & 20 & 20 \\
\hline
\end{tabular}

Fonte: Tabela construída pelos autores.

Em geral, as maiores porcentagens de erros ocorreram pela escolha das comparações onde as frações apresentaram os menores valores numéricos no denominador da fração. Este fato ocorreu em oito das 14 relações testadas no GEQ (D1A1, D2A2, B1D1, B2D2, C1D1, C3D3, D2C2 e D3C3), em dez no GIP (D1A1, D2A2, B1D1, D1B1, D2B2, D3B3, C1D1, D1C1, D2C2 e D3C3) e em sete no GIPEQ (D1A1, B1D1, D1B1, D2B2, C1D1, C3D3 e D2C2). Cabe ressaltar que nas relações D1A1, B1D1, C1D1 e D2C2 este fato ocorreu nos três grupos experimentais. Maiores porcentagens de erros pela escolha das comparações onde as frações apresentaram os maiores valores numéricos no denominador da fração ocorreram em quatro relações no GEQ (D1B1, D3B3, C2D2 e D1C1), em cinco no GIPEQ (D2A2, B2D2, D3B3, C2D2 e D1C1) e em apenas uma no GIP (C3D3).

\section{DISCUSSÃO}

Os objetivos do presente experimento foram investigar o efeito (1) do treino com instruções programadas sobre como resolver problemas fracionários, (2) do treino de relações condicionais entre estímulos pictóricos e numéricos e entre estímulos numéricos e numéricos segundo o paradigma proposto por Sidman e Taiby, (1982) e (3) do treino conjunto entre instruções programadas e treino de relações condicionais sobre: a) formação classes de equivalência de estímulos; b) a expansão das classes de equivalência de estímulos formadas e a generalização para novas situações e c) a resolução de problemas fracionários do tipo lápis e papel. A ocorrência de maiores porcentagens de acertos em problemas fracionários expressos de forma absoluta (condição $\mathrm{AB}$ ) do que em problemas expressos de forma relativa (condições $\mathrm{BC}$ e $\mathrm{AD}$ ) replicaram dados de pesquisas anteriores (CARRAHER; SHLIEMANN, 1992; SANTOS, 1999) quanto à dificuldade dos alunos em compreender o conceito de magnitude relativa entre frações.

Nos testes de simetria e transitividade/equivalência 13 participantes demonstraram a emergência de relações não ensinadas diretamente e corroboraram dados da literatura sobre a emergência de classes de estímulos a partir do treino direto de apenas algumas relações 


\section{(2tinnerarius Refiectionis:- =

condicionais (SIDMAN; TAIBY, 1982; DE ROSE, KATO, THÉ; KLEDARAS, 1997). Podese dizer que eles formaram as classes $\mathrm{A} 1 \mathrm{~B} 1 \mathrm{C} 1, \mathrm{~A} 2 \mathrm{~B} 2 \mathrm{C} 2$ e $\mathrm{A} 3 \mathrm{~B} 3 \mathrm{C} 3$, sendo um estímulo pictórico e dois estímulos fracionários (um expresso de forma absoluta e outro de forma relativa).

Os desempenhos decrescentes observados ao longo dos blocos de testes de expansão das classes para 13 dos 15 participantes estão em desacordo com o a literatura da área (DE ROSE, et al, 1997) e permitem questionar se realmente ocorreu a aquisição do conceito de magnitude relativa entre frações para esses participantes. Uma possível explicação para esse fato pode ser a natureza relativa das frações presentes nas relações testadas (DA, BD, DB, CD e DC), o que segundo a literatura representa um ponto de dificuldade de aprendizagem para os alunos (CARRAHER; SHLIEMANN, 1992; SANTOS 1999). É possível ainda que os treinos programados no presente trabalho tenham gerado apenas relações arbitrárias entre os estímulos. Assim, a formação das três classes de estímulos pode ter ocorrido devido ao efeito direto do procedimento de pareamento com o modelo, o qual, segundo a literatura é uma forma eficiente para estabelecer relações entre estímulos mesmo quando eles são dissimilares (DE ROSE, 1993).

Os dados do presente experimento indicam que o treino com instrução programada foi uma estratégia tão eficiente quanto o treino de pareamento com o modelo para estabelecer relações condicionais entre estímulos pictóricos e numéricos e entre estímulos numéricos e numéricos. De forma geral, os desempenhos observados entre os participantes que fizeram e os que não fizeram esse tipo de treino foram semelhantes em todas as condições experimentais. Contudo, no teste do tipo lápis e papel com problemas expressos de forma relativa os maiores ganhos $(69 \%)$ ocorreram para os participantes que fizeram tanto o treino com instruções programadas quanto o treino de relações condicionais (GIPEQ). Esse resultado encontra respaldo tanto na literatura sobre ensino com instrução programada (SKINNER, 1972) quanto no de equivalência de estímulos (SIDMAN; CRESSON, 1973; STROMER, MACKAY; STODDARD, 1992). Um ponto comum entre as duas propostas de ensino é o cuidado em assegurar que o aprendiz adquira respostas consideradas como prérequisitos ou precorrentes para comportamentos posteriores e aumentar as chances de se obter sucesso na execução da tarefa (BAUM, 1999). Os treinos com instrução programada ensinaram aos participantes como identificar as representações fracionárias apresentadas nos 


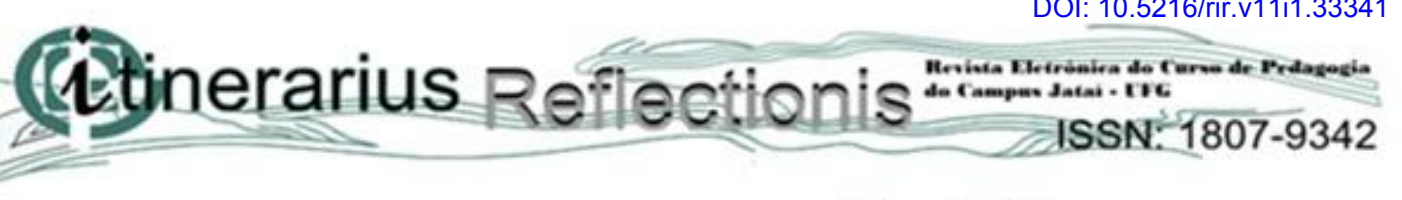

$$
\text { v.11, n. } 1,2015
$$

problemas, como simplificá-las (dividindo ou multiplicando) e como escrever a nova resposta (fração), habilidades que são essenciais para a resolução de problemas que requeiram julgamento proporcional, razão e o conceito de magnitude relativa entre frações. Ainda, o treino com instruções programadas apresentou de forma gradual as instruções constantes na regra de três. Segundo Albuquerque e Ferreira (2001), a extensão da regra pode ser uma variável inversa à probabilidade dela ser seguida, ou seja, quanto maior a extensão da regra menor a probabilidade dela controlar o comportamento do ouvinte. Assim, uma regra que descreve um número extenso de respostas pode dificultar o controle individual de cada uma delas e tornar o encadeamento necessário para a emissão da resposta solução também mais difícil. Uma possibilidade para estudos futuros quanto ao efeito das instruções é aumentar o número de passos apresentados durante o treino com instruções programadas na resolução dos exercícios ou aumentar o número de exemplos disponibilizados durante o treino.

Os maiores escores observados no teste de generalização 1 (exceto P2) podem ser explicado pela possibilidade dos participantes resolverem os problemas aplicando o referencial de metade (SPINILLO, 1992), ou seja, pela divisão ou multiplicação dos numeradores e/ou denominadores das frações pelo número 2.

Aparentemente, os resultados mostraram inconsistência em relação ao efeito que o treino com instruções programadas teve sobre o desempenho no teste de expansão das classes formadas e os ganhos no teste de avaliação final no grupo GIPEC, uma vez que nesse grupo ocorreram os menores escores no primeiro teste e os maiores ganhos no segundo teste. Contudo, há que se considerar a natureza diferente das duas tarefas programadas para cada condição específica de teste. No teste de expansão, a tarefa seguiu o procedimento de pareamento com o modelo apresentados em pastas e no segundo uma tarefa do tipo lápis e papel, a qual se aproxima mais das atividades rotineiras do ensino regular.

Os resultados mostraram que as maiores porcentagens de erros no teste D-(ABC) ocorreram pela escolha das comparações onde as frações apresentaram os menores valores numéricos no denominador da fração. Esses dados indicam que esses erros estiveram mais sob o controle do denominador da fração, neste caso o denominador com o menor valor numérico, do que da relação existente entre o numerador e o denominador. Segundo Spinillo (1993), levar em consideração a relação parte-todo, ou seja, a relação entre numerador e denominador, é uma condição imprescindível para a compreensão do conceito de proporção. 


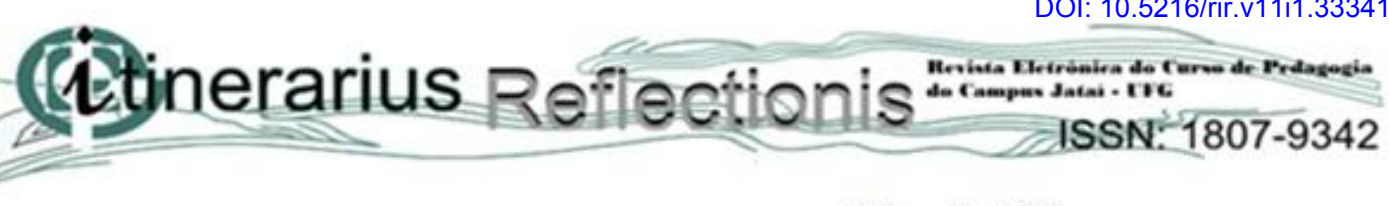

$$
\text { v.11, n. } 1,2015
$$

Assim, a possível ausência dessa resposta relacional explicaria as escolhas dos estímulos de comparação incorretos ao longo dos testes realizados no presente estudo. Outra possível explicação para a escolha das comparações incorretas com os menores valores nos denominadores das frações pode ser o fato de que, em geral, nessas comparações os denominadores eram mais facilmente divididos por dois, o que aumentou a possibilidade dos participantes tentarem resolver os problemas, de foram indevida, a partir do referencial de metade (SPINILLO,1992).

Quanto aos objetivos propostos para o presente estudo pode-se concluir que: a) ocorreu a formação de classes de equivalência de estímulos em todas as condições experimentais (GIP, GIPEQ e GEQ), b) as porcentagens de acertos diminuíram ao longo dos blocos de tentativas na condição de teste de expansão das classes para todos os grupos e houve maior generalização da resposta no primeiro teste, e c) a interação entre treino com instruções e equivalência de estímulos produziu maiores porcentagens de acertos na resolução de problemas do tipo lápis e papel.

Certamente, o ensino do conceito de frações é um desafio para os educadores e, conforme sugeriu Teixeira (2006), estudar o papel de instruções, pode ser um caminho interessante para encontrar respostas a esse problema, visto que, segundo ela, um conjunto arbitrário de regras lógicas tem para o comportamento matemático a mesma importância que um conjunto de regras gramaticais tem para o comportamento verbal oral e escrito.

\section{REFERÊNCIAS}

ALBUQUERQUE, L. C.; FERREIRA, K. V. D. Efeitos de regras com diferentes extensões sobre o comportamento humano. Psicologia: reflexão e crítica, 14 (1), 143-155, 2001.

BAUM, W. M. Compreender o behaviorismo: ciência, comportamento e cultura. Porto Alegre: ArtMed, 1999.

CARMO, J. S. O conceito de número colo rede de relações. In: R. Kerbauy (org). Sobre comportamento e cognição, Santo André, SP: Esetec Editores Associados vol. 5, p. 97-113, 2000.

CARRAHER, D. W.; SCHLIEMANN, A. D. A compreensão de frações como magnitudes relativas. Psicologia: Teoria e Pesquisa, 8(1), 67-78, 1992. 


\section{(titinerarius Reflectionis $=$}

CARRAHER, T. N., CARRAHER, D. W., SCHLIEMANN, A. D.; RUIZ, E. R. L. Proporcionalidade na educação científica e matemática: quantidades medidas por razões. Revista Brasileira de Estudos Pedagógicos, 67, 93-107, 1986.

CARRAHER, T. N., CARRAHER, D. W.; SCHLIEMANN, A. D. Na vida dez, na escola zero: os contextos culturais da aprendizagem matemática. In: Carraher, T. N., Carraher, D. W.; Schliemann, A. D. (ORG.). Na vida dez, na escola zero. São Paulo: Cortez, pp. 23-43, 1993.

DE ROSE, J. C. Classes de estímulos: implicações para uma análise comportamental da cognição. Psicologia: Teoria e Pesquisa, 9 (2), 283-303, 1993.

Prefácio. En: J. S. Carmo e P. S. T. Prado (orgs). Relações simbólicas e aprendizagem da matemática. pp 49-68. Santo André: ESETec Editores Associados, 2010.

DE ROSE, J. C., KATO, O. M., THÉ, A. P.; KLEDARAS, J. B. Variáveis que afetam a formação de classes de estímulos: estudos sobre efeitos do arranjo de treino. Acta Comportamentalia, 5, 143-163. 1997.

LINCH, D. C.; CUVO, A. J. Stimulus equivalence instruction of fraction-decimal relations. Journal of Applied Behavior Analysis, 28, 115-126, 1995.

LOPES, A. J. O que nossos alunos podem estar deixando de aprender sobre frações, quando tentamos lhes ensinar frações. Bolema, 21(31), 1-22, 2008.

SANTOS, A. C. G. Equivalência de frações: como anda a compreensão dos alunos?. Estudos: Revista da Universidade Católica de Goiás, 26,(2), 233-249, 1999.

SANTOS, A. C. G.; BAY, C. K.; SOUZA, A. V. Aprendizagem do conceito de proporção: equivalência de estímulos e controle instrucional. Trabalho apresentado na XXVII Reunião anual de Psicologia da Sociedade Brasileira de Psicologia, Ribeirão Preto, SP, 1997.

SANTOS, A. C. G.; CAMESCHI, C.; HANNA, E. S. Ensino de frações baseado no paradigma de equivalência de estímulos. Revista Brasileira de Análise do Comportamento, 5, (1), 19-4, 2009.

SAUNDERS, R. R. WACHTER, J.; SPRADLIN, J. E. Establishing auditory stimulus control over an eight-member equivalence class via conditional discrimination procedure. Journal of the Experimental Analysis of Behavior, 49, 95-115, 1988.

SIDMAN, M. KIRK, B.; WILSON-MORRIS, M. Six-member stimulus classes generated by conditional-discrimination procedures. Journal of the Experimental Analysis of Behavior, 43, 21-42, 1985. 


$$
\text { v.11, n. } 1,2015
$$

in severe retardation. Equivalence relations and behavior: a research story. Author Cooperative, Inc: Boston, Massachusets, 1973.

SIDMAN, M.; TAILBY, W. Conditional discrimination vs. matching to sample: an expansion of the testing paradigm. Journal of the Experimental Analysis of Behavior, 37, 5-22, 1982.

SKINNER, B. F. Questões recentes na análise comportamental. Tradução de Anita Liberalesso Néri. Campinas: Papirus, 1991.

SILVA, M. J. F.; ALMOULOUD, A. S. As operações com números racionais e seu significados a partir da concepção parte-todo. Bolema, 21(31), 55-78, 2008.

Tecnologia do ensino. Tradução de Rodolpho Azzi. São Paulo: EPU, 1972.

SPINILlo, A. G. A importância do referencial de metade e o desenvolvimento do conceito de proporção. Psicologia: Teoria e Pesquisa, 8(3), 305-317, 1992.

. As relações de primeira ordem em tarefas de proporção: uma outra explicação quanto às dificuldades das crianças. Psicologia: Teoria e Pesquisa, 9(2), 349-364, 1993.

STROMER, R., MACKAY, H. A.; STODDARD, L. T. Classroom applications of stimulus equivalence technology. Journal of Behavioral Education, 2(3), 225-256, 1992.

TEIXEIRA, A. M. S. Análise de contingências em programação de ensino infantil: liberdade e efetividade na educação. Santo André, SP: ESETec, 2006.

TINOCO, L. A. A.; LOPES, M. L. Frações: dos resultados de pesquisa à prática em sala de aula. Revista Brasileira de Educação Matemática, 2, pp 13-18, 1994. 